\title{
Rhinogenic Orbital Inflammation - What Has Changed over the Past 50 Years?
}

\author{
Viktor Chrobok ${ }^{1}$, Arnošt Pellant ${ }^{3}$, Petra Mandysová4, Jan Mejzlík , Jana Dědková2, Petr Čelakovský1,*
}

\begin{abstract}
Introduction: The purpose of the study was to compare the incidence, diagnostics, and treatment of rhinogenic inflammatory complications over the past 50 years.

Material and methods: Retrospective study of 292 patients of ENT department, University hospital: Group A treated from 1966 to 1995 , Group B treated from 1996 to 2015.

Results: Preseptal inflammation was the most common type (73\% vs. $74 \%)$, followed by subperiosteal abscess (21\% vs. $20 \%)$. Surgery was indicated in $35 \%$ vs. $37 \%$ of the patients $(p=0.434)$. The most commonly used surgical approach was the external route ( $80 \%)$ in Group $A$ and endoscopic endonasal surgery $(60 \%)$ or a combination of endoscopic surgery of the paranasal sinuses and external orbitotomy (30\%) in Group B $(p<0.001)$. The percentage of reoperations was $13 \%$ vs. $14 \%$. In cases of revision surgery, the orbit was always treated using the external surgical approach. Complete recovery was achieved in $92 \%$ and $98.5 \%$ of the patients belonging to Group A and B, respectively $(p=0.622)$.

Conclusion: Nowadays, the endoscopic endonasal approach is the most frequently used surgical technique for paranasal sinuses. The technique used to treat the orbital complication itself depends on several factors. Nowadays, the endoscopic approach is preferred. The external approach can be considered in the case of recurrent or persistent abscesses, especially if they are located in the upper or the lateral part of the orbit.
\end{abstract}

\section{KEYWORDS}

sinusitis; endoscopic surgery; orbital inflammation

\section{AUTHOR AFFILIATIONS}

${ }^{1}$ Department of Otorhinolaryngology and Head and Neck Surgery, University Hospital Hradec Králové, Charles University, Faculty of Medicine in Hradec Králové, Hradec Králové, Czech Republic

2 Department of Radiology, University Hospital Hradec Králové, Hradec Králové, Czech Republic

${ }^{3}$ Department of Otorhinolaryngology and Head and Neck Surgery, Pardubice Regional Hospital, Faculty of Health Studies, University of Pardubice, Pardubice, Czech Republic

${ }^{4}$ Faculty of Health Studies, University of Pardubice, Pardubice, Czech Republic

* Corresponding author: Department of Otorhinolaryngology and Head and Neck Surgery, University Hospital Hradec Králové, Sokolská 581, 50005 Hradec Králové, Czech Republic; e-mail: petr.celakovsky@fnhk.cz

Received: 10 July 2019

Accepted: 22 August 2019

Published online: 30 October 2019

Acta Medica (Hradec Králové) 2019; 62(3): 94-98

https://doi.org/10.14712/18059694.2019.131

(c) 2019 The Authors. This is an open-access article distributed under the terms of the Creative Commons Attribution License (http://creativecommons.org/licenses/by/4.0), which permits unrestricted use, distribution, and reproduction in any medium, provided the original author and source are credited. 


\section{INTRODUCTION}

Inflammatory conditions affecting the orbit (orbital cellulitis) represent - from the aetiological point of view a relatively broad spectrum of conditions. Primary orbital inflammation is rare; it is presumably preceded by an injury that penetrates orbital tissues. Secondary orbital inflammation is more common; it develops due to spread of infection from the surrounding structures. In particular, this concerns cases of rhinosinusitis. However, inflammation of the lacrimal pathways or the teeth (i.e. odontogenic inflammation) or inflammation arising from the surrounding skin (erysipelas or a furuncle involving the nose or lip), i.e. dermatogenic infection, can spread to the orbit as well. Only rarely does orbital inflammation spread from a distal infection site, via the intracranial or the haematogenous route.

Rhinogenic orbital complications are the most frequent. Pansinusitis predominates in comparison with isolated inflammation of the paranasal sinuses (60-80\%). In descending order, the following types of inflammation can be listed: inflammation of the ethmoidal, frontal, maxillary, and sphenoid sinuses. Rhinogenic orbital inflammation mainly affects children aged 5 to 10 years. In older children or adults, complications arising from inflammation of the frontal sinuses occur relatively more frequently (1). Typically, the clinical course is more favourable in children (preseptal inflammation predominates). In contrast, adults have a higher risk of developing postseptal inflammatory complications and persistent vision handicap (2). Furthermore, aetiological agents in children are different than in adults. In childhood, the principal causative agents are Streptococcus pneumoniae, nontypeable Haemophilus influenza, and Moraxella catarrhalis, whereas in adults, polymicrobial inflammation characterized by the presence of anaerobes is the most common type. The microbiology of infections of the paranasal sinuses can be anticipated according to the patient's age, clinical presentation, nasal patency and immunocompetence $(3,4)$. Although patients seek medical assistance when experiencing acute symptoms, histological findings in the mucous membranes of the paranasal sinuses in surgically treated patients indicate that in these cases, chronic inflammatory infiltration prevails. Therefore, from the aetiological point of view, acute exacerbation of chronic rhinosinusitis is frequently involved in the development of orbital cellulitis.

The classification of rhinogenic inflammatory complications involving the orbit is based on Chandler's classification (5-7) (Table 1).

The objective of our study was to compare the incidence, diagnosis, and treatment of rhinogenic inflammatory complications over the past 50 years.

Tab. 1 Classification of orbital inflammations according to Chandler.

\begin{tabular}{|l|l|}
\hline Stage 1 & Preseptal cellulitis \\
\hline Stage 2 & Orbital cellulitis \\
\hline Stage 3 & Subperiosteal abscess \\
\hline Stage 4 & Orbital abscess \\
\hline Stage 5 & Cavernous sinus thrombosis \\
\hline
\end{tabular}

\section{MATERIALS AND METHODS}

This was a retrospective study of 292 patients with rhinogenic orbital inflammation, hospitalized in the Department of Otorhinolaryngology and Head and Neck Surgery of the University Hospital in Hradec Kralove over the past 50 years (between January 1, 1966 and December 31, 2015). The study patients were divided into two groups. The first group (Group A) consisted of patients treated between 1966 and 1995, i.e. when functional endonasal surgery was not yet a standard treatment in the above-mentioned workplace. The second group (Group B) consisted of patients treated between 1996 and 2015; functional endonasal surgery was a standard surgical method. Table 2 presents patient characteristics of both groups. In addition to basic demographic data, the following factors were evaluated in both groups: the type (stage) of inflammatory complication involving the orbit, the type of treatment (conservative or surgical), the selected surgical approach (endoscopic or external), the treatment outcome, and other complications.

Statistical analysis: For the statistical analysis the Statistica (data analysis software system), version 13 was used. Fisher exact test was used for estimation of statistical significance between the groups and $p$-values less than 0.05 were considered as statistically significant.

Tab. 2 Patient characteristics.

\begin{tabular}{|l|l|l|}
\hline Item & $\begin{array}{l}\text { Group A } \\
(\mathbf{1 9 6 6 - 1 9 9 5 )}\end{array}$ & $\begin{array}{l}\text { Group B } \\
(\mathbf{1 9 9 6 - 2 0 1 5 )}\end{array}$ \\
\hline Number of patients & 159 & 133 \\
\hline Cases per year & 5.3 & 6.7 \\
\hline Male $:$ female & $2: 1$ & $3: 1$ \\
\hline $\begin{array}{l}\text { Children and Teenagers } \\
\text { ( } \leq 20 \text { years old) }\end{array}$ & $121(76 \%)$ & $81(61 \%)$ \\
\hline
\end{tabular}

\section{RESULTS}

\section{THE TYPE OF ORBITAL COMPLICATION}

In both groups, the proportion of individual types of orbital complications was similar. Preseptal inflammation prevailed (73\% vs. $74 \%$ ); subperiosteal abscess was the second most common type ( $21 \%$ vs. $20 \%$ ). In both groups, only rarely were patients diagnosed as having postseptal complications (orbital cellulitis, abscess - Table 3). As for unusual associated intracranial complications, we observed three cases of cavernous sinus thrombosis in patients belonging to Group A, and two cases of epidural abscess in Group B.

Tab. 3 Distribution of orbital complications.

\begin{tabular}{|l|l|l|}
\hline Orbital complication & Group A & Group B \\
\hline Preseptal cellulitis, abscess & $119(75 \%)$ & $98(74 \%)$ \\
\hline Subperiosteal abscess & $33(21 \%)$ & $27(20 \%)$ \\
\hline Orbital cellulitis, abscess & $6(4 \%)$ & $5(4 \%)$ \\
\hline Subperiosteal and orbital abscess & $1(0.6 \%)$ & $3(2 \%)$ \\
\hline Associated complications & $\begin{array}{l}3 \text { (cavernous } \\
\text { sinus } \\
\text { thrombosis) }\end{array}$ & $\begin{array}{l}2 \text { (epidural } \\
\text { abscess) }\end{array}$ \\
\hline
\end{tabular}


CONSERVATIVE TREATMENT VERSUS SURGERY

In both groups, most patients were treated conservatively; surgery was indicated in approximately one-third of the patients ( $35 \%$ vs. $37 \%$ ). Statistically, there was no difference in treatment strategy between the Group A and Group B $(p=0.434)$.

\section{SURGICAL APPROACH}

A comparison of the surgical approaches revealed noteworthy differences (Table 4). In Group A, the external route constituted the most commonly used surgical approach (80\%) whereas most patients belonging to Group $B$ underwent endoscopic endonasal surgery (60\%) or a combination of endoscopic surgery of the paranasal sinuses and external orbitotomy (30\%). This difference in number of endoscopical and external surgical approaches was statistically significant $(p<0.001)$. In both groups, the percentage of reoperations was similar (13\% vs. $14 \%)$. In most cases, reoperation was indicated for persistent orbital abscesses, localized mainly in the superolateral part of the orbit. In cases of revision surgery, the orbit was always treated using the external surgical approach.

Tab. 4 Treatment.

\begin{tabular}{|l|l|l|}
\hline Treatment & Group A & Group B \\
\hline Conservative & $103(65 \%)$ & $84(63 \%)$ \\
\hline Surgical & $56(35 \%)$ & $49(37 \%)$ \\
\hline - external approach & $44(80 \%)$ & $5(10 \%)$ \\
\hline - endoscopic & $6(10 \%)$ & $29(60 \%)$ \\
\hline - combination & $6(10 \%)$ & $15(30 \%)$ \\
\hline - reoperation & $7(13 \%)$ & $7(14 \%)$ \\
\hline
\end{tabular}

\section{COMPLICATIONS}

Complete recovery was achieved in $92 \%$ of the patients belonging to Group A and $98.5 \%$ of the patients belonging to Group B (Table 5). Diplopia was the most common permanent consequence following resolution of inflammation ( $3.8 \%$ vs. $1.5 \%$ ). In Group B, there were no cases of residual visual impairment or death, whereas in Group A, mild visual impairment, unilateral blindness, and death were noted in $2.5 \%, 1.3 \%$, and $0.6 \%$ of the patients, respectively. In spite of this fact, there was no difference in complications rates between the Group A and Group B on statistical analysis $(p=0.622)$. Complications as visual impairment, blindness and death were typical just only for adults.

Tab. 5 Results of treatment.

\begin{tabular}{|l|l|l|}
\hline Treatment Result & Group A & Group B \\
\hline Healed & $146(91.8 \%)$ & $131(98.5 \%)$ \\
\hline Impaired vision & $4(2.5 \%)$ & 0 \\
\hline Blindness & $2(1.3 \%)$ & 0 \\
\hline Diplopia & $6(3.8 \%)$ & $2(1.5 \%)$ \\
\hline Death & $1(0.6 \%)$ & 0 \\
\hline
\end{tabular}

\section{DISCUSSION}

\section{CLINICAL TERMINOLOGY OF RHINOGENIC ORBITAL INFLAMMATION}

Differences between inflammation forms are expressed through the terms abscess, phlegmon, and cellulitis. Abscess is a collection of purulent material, typically due to inflammation, that is contained in a pathological cavity. Phlegmon is a diffuse, purulent inflammatory process spreading through sparse tissues. Cellulitis is a well-demarcated inflammatory response in the soft tissue with an accumulation of inflammatory cells. In the case of orbital cellulitis, there is a well-demarcated response in the soft tissue with an accumulation of inflammatory cells in a certain zone from the periorbita, towards the inside of the orbital cavity. Orbital phlegmon is an inflammation of the soft tissues of the entire eye socket. It is a diffuse bacterial inflammation that can be associated with orbital venous thrombosis together with septic signs and symptoms (8-11). The terms preseptal, epiperiorbital (subperiosteal), and the orbital space itself are used to describe the location of the inflammation in the orbit. The term preseptal cellulitis is preferred over the term inflammatory eyelid oedema because it is a better way of conveying the location of the inflammation, anterior to the orbital septum. The term subperiosteal is the most frequently used term for an abscess that is located between the bone and the periosteum. However, the term epiperiorbital is more comprehensible as it clearly describes the location, outside the periorbita. The terminology is similar to that used to describe intracranial complications, where the term epidural represents the space outside the cranial dura mater, i.e. between the dura mater and the bone. The anatomic boundary of the periorbita enables classification of inflammatory orbital complications into phase I and phase II complications (again, the terminology is analogous to that used to describe intracranial complications, with the cranial dura mater being the analogous boundary). Phase I complications include preseptal cellulitis and subperiosteal abscess; phase II complications include orbital cellulitis and orbital abscess (12).

As for our study sample, the proportion of individual types of orbital complications remained essentially the same in both groups during the study period. Preseptal inflammation prevailed; subperiosteal abscess was the second most common type, and postseptal complications were rare. It is interesting to compare our study sample with that from Graz (13), which, by contrast, consisted of a high percentage of patients with postseptal complications (orbital abscess in 43\%). This finding could probably be explained by a higher concentration of patients with serious postseptal complications in the workplace in Graz.

\section{DIAGNOSTICS OF RHINOGENIC ORBITAL INFLAMMATION}

The diagnosis of inflammatory orbital complications includes medical history taking, an ENT examination using nasal endoscopy, and an eye examination. Imaging-based examination is an indispensable method that enables a de- 
tailed assessment of the condition of the paranasal sinuses as well as the orbital inflammation itself.

If the patient has preseptal cellulitis and his or her clinical condition is good, it is possible to wait and see whether imaging-based examination will be needed. Currently, plain X-rays are not indicated; they are not beneficial in orbital complications of sinusitis. In adult patients, a CT scan (ideally, using a contrast agent) is the method of first choice (Fig. 1). Noncontrast or contrast-agent enhanced MR imaging is preferred in paediatric patients (Fig. 2) as well as if postseptal orbital complication, cavernous sinus thrombosis, or another current intracranial complication is suspected (14). Unequivocal indications for imaging-based examination include the following conditions: inability to accurately evaluate vision, marked protrusion of the eyeball, ophthalmoplegia, bilateral oedema, visual

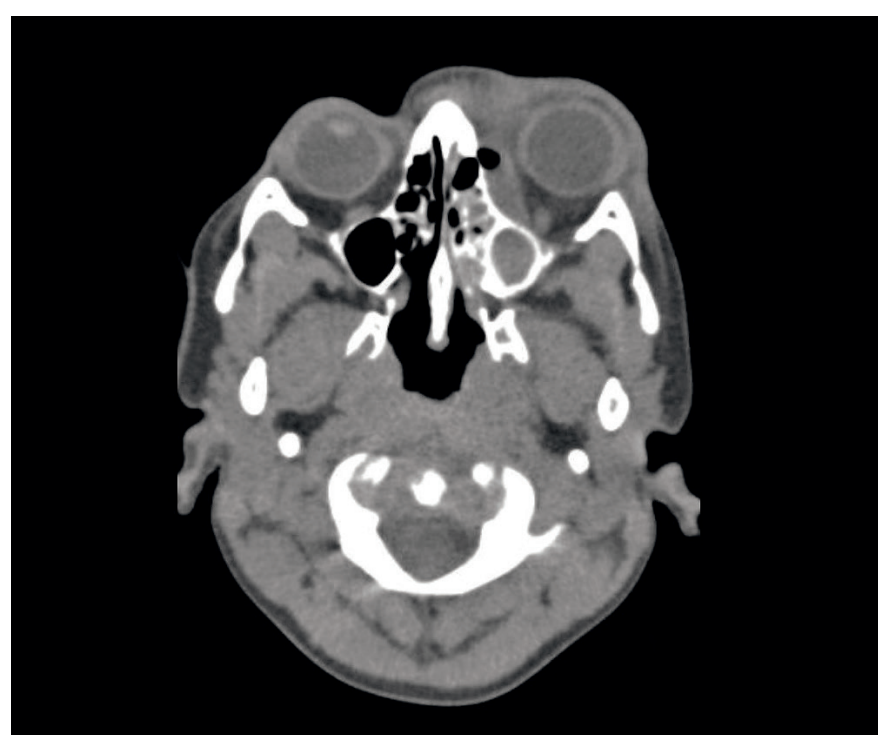

Fig. 1 CT, horizontal section: left-sided pansinusitis, defect of lamina papyracea, inflammatory infiltration with air bubble along medial orbital wall.

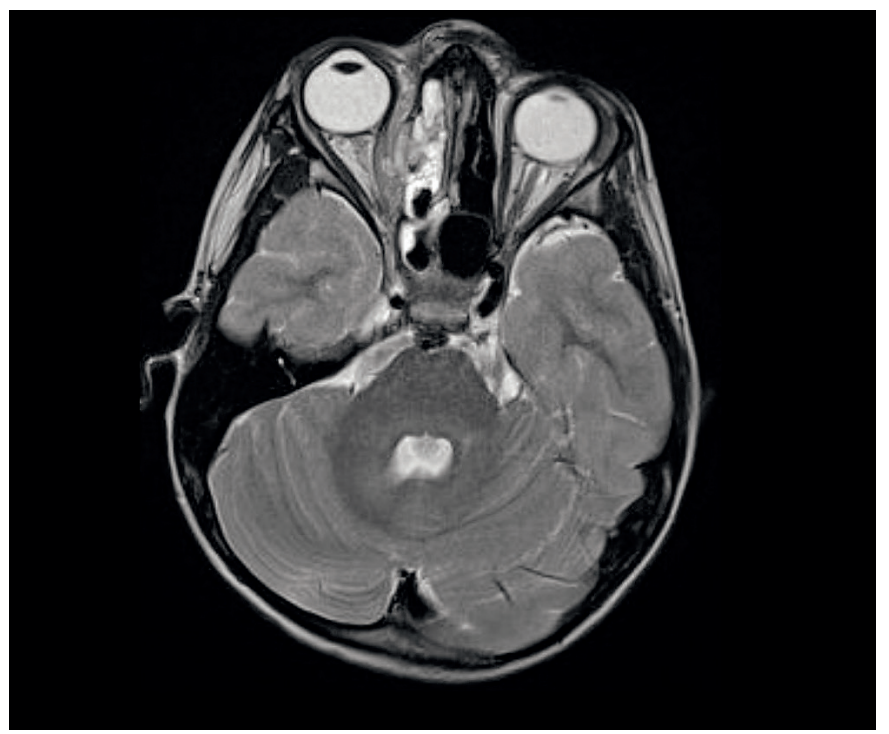

Fig. 2 MR, horizontal section: inflammatory infiltration of ethmoids and medial part of orbit on the right with dislocation of the course of medial rectus muscle. disturbances, no improvement in health status after 24 hours of intensive conservative treatment using intravenous antibiotics, and CNS signs and symptoms.

\section{TREATMENT OF RHINOGENIC ORBITAL INFLAMMATION \\ Conservative management}

Antibiotic therapy is the mainstay of conservative management of orbital inflammation; broad-spectrum antibiotics are used. Monotherapy (aminopenicillin or cephalosporin) is sufficient for the treatment of preseptal inflammation, especially in children. By contrast, postseptal inflammation and orbital cellulitis in adults may require the use of combination therapy with two antibiotics (the addition of metronidazole or clindamycin) as in such cases, anaerobic strains are more frequently represented. In the first phase, empirical antibiotic therapy is instituted, and based on the culture and sensitivity results, the therapy can be adjusted according to further progress. Nasal mucosa toilet facilitates unobstructed passage through the nasal cavity as well as the unblocking of the exit points of the paranasal sinuses. Systemic or local corticosteroids can be used (9) although there are conflicting opinions egarding the question whether or not they are indicated for treatment of acute inflammation. As part of conservative management, maxillary sinus puncture can help in orbital cellulitis that develops due to maxillary sinusitis of odontogenic origin.

Surgical management

Surgical management - without any delay - is undeniably indicated for the treatment of subperiosteal and orbital abscess. In some patients (especially in those presenting with anaerobic infections), an imaging-based examination reveals air bubbles in the subperiosteal space, or in rare cases, in the intraconal space. This finding frequently precedes the development of a well-demarcated abscess area, which is an indication for surgical intervention as well (the surgical procedure frequently shows purulent collections in the corresponding area). Furthermore, a CT scan may not reveal orbital abscess in all patients, especially if it is performed without a contrast agent. Therefore, even if an imaging-based examination does not reveal any abscess area, it is necessary to consider a surgical intervention (so that, at the very least, the primary inflammation site in the paranasal sinuses could be treated and the epiperiorbital space could be explored) in the following conditions: impaired visual acuity, progression of local and systemic signs and symptoms despite adequate conservative management for more than 24 hours, or no local improvement after 48-72 hours of antibiotic therapy.

Currently, it is possible to use the following surgical approaches:

The endoscopic endonasal approach - treatment of inflammation in the area of the affected paranasal sinuses; ethmoidectomy is used the most frequently (15). Following entry via the lamina papyracea, it is possible to drain subperiosteal abscess, which is in close contact with the medial wall of the orbit. All abscesses that do not meet the 
mentioned requirement and that are located deeper within the orbit are less suitable for the endonasal approach. Treatment of orbital abscess entails making an incision in the periorbita. The endonasal approach requires excellent surgical skilfulness. The endonasal approach is affected by bleeding from the inflamed mucosa of the paranasal sinuses and by small spaces in paediatric patients.

The external approach - it consists of orbitotomy (usually medial), external ethmoidectomy and drainage of subperiosteal or orbital abscess. Treatment of orbital abscess entails making an incision in the periorbita. The external approach is recommended if an abscess is located in the eyelid or in the upper, the lower, and the lateral part of the orbit as well as in patients undergoing revision surgery following endoscopic endonasal treatment.

The combined approach - endoscopic endonasal approach for paranasal sinuses and external approach for inflammatory orbital complications.

In our study sample, a large proportion of the patients received conservative treatment; surgical treatment was indicated in approximately one-third of the patients. In Group A, the external surgical approach was the most common technique, whereas Group B was treated mainly endoscopically or by using a combination of endoscopic surgery of the paranasal sinuses and external orbitotomy. The percentage of reoperations was similar in both groups; the advantages of the endoscopic approach include reduced perioperative morbidity and no scars in the facial area. Nowadays, the endoscopic approach is preferred. The external approach can be considered in the case of recurrent or persistent abscesses, especially if they are located in the upper or the lateral part of the orbit.

\section{CONCLUSIONS}

Nowadays, the endoscopic endonasal approach is the most frequently used surgical technique for paranasal sinuses. The technique used to treat the orbital complication itself depends on several factors. Nowadays, the endoscopic approach is preferred. The external approach can be considered in the case of recurrent or persistent abscesses, especially if they are located in the upper or the lateral part of the orbit.

\section{ACKNOWLEDGEMENTS}

This article was supported by the project (Ministry of Health, Czech Republic) for conceptual development of research organization 00179906.

\section{DISCLOSURE STATEMENT}

The authors declare that they have no competing interest.

\section{REFERENCES}

1. Celakovsky P, Vokurka J, Drsata J, Ruzicka J. Management of the orbital complications of sinusitis in children. Head Neck Diseas 2003; 12: 33-7.

2. Celakovsky P, Vokurka J, Skoloudik L, Ruzicka J. Optic neuritis and paranasal sinus diseases. Cent Eur J Med 2011; 6: 117-9.

3. Wald ER. Microbiology of acute and chronic sinusitis in children and adults. Am J Med Sci 1998; 316: 13-20.

4. Skoloudik L, Vokurka J, Zborayova K, Celakovsky P, Kucera M, Ryska A. Cytology of the nasal mucosa after total laryngectomy. Acta Otolaryngol 2009; 129: 1262-5.

5. Georgalas C, Fokkens W. Rhinology and skull base surgery. Stuttgart: Thieme; 2013.

6. Chandler JR, Langenbrunner DJ, Stevens ER. The pathogenesis of orbital complications in acute sinusitis. Laryngoscope 1970; 80: $1414-28$

7. Mortimore S, Wormald PJ. The Groote Schuur hospital classification of the orbital complications of sinusitis. J Laryngol Otol 1997; 111: 719-23.

8. Healy GB. The pathogenesis of orbital complications in acute sinusitis. Laryngoscope 1997; 107: 441-6.

9. Chrobok V, Hybasek I, Pellant A. Rhinogenic inflammatory orbital complications in the materials of the ENT clinic in Hradec Kralove. Otorinolaryng a Foniat (Prague) 1994; 43: 128-31.

10. Chrobok V, Vokurka J, Hybasek I, Pellant A. Rhinogenic inflammatory orbital complications in the materials of the ENT clinic in Hradec Králové in 1966-1995. Central East Europ J Otorhinolaryngol Head Neck Surg 1996; 1:313-6.

11. Chrobok V, Vokurka J. Contribution to clinical anatomy, pathophysiology and treatment of rhinogenic orbital inflammations. Otorinolaryng a Foniat (Prague) 1998; 47: 3-8.

12. Pellant A. Orbital complications of sinusitis. Otorinolaryng a Foniat (Prague) 1973; 22: 283-6.

13. Teinzer F, Stammberger H, Tomazic PV. Transnasal endoscopic treatment of orbital complications of acute sinusitis: the Graz concept. Ann Otol Rhinol Laryngol 2015; 124: 368-73.

14. Kennedy DW, Hwang PH. Rhinology: Diseases of the nose, sinuses and skull base. New York: Thieme; 2012.

15. Page EL, Wiatrak BJ. Endoscopic vs external drainage of orbital subperiosteal abscess. Arch Otolaryngol Head Neck Surg 1996; 122: 737-40. 